\title{
Discrete and Conservative Factorizations in Fib(B)
}

\author{
Alan S. Cigoli ${ }^{1} \cdot$ Sandra Mantovani ${ }^{2} \cdot{\text { Giuseppe } \text { Metere }^{3}}$ \\ Received: 27 January 2020 / Accepted: 20 October 2020 / Published online: 4 November 2020 \\ (c) The Author(s) 2020
}

\begin{abstract}
We focus on the transfer of some known orthogonal factorization systems from Cat to the 2-category $\mathrm{Fib}(B)$ of fibrations over a fixed base category $B$ : the internal version of the comprehensive factorization, and the factorization systems given by (sequence of coidentifiers, discrete morphism) and (sequence of coinverters, conservative morphism) respectively. For the class of fibrewise opfibrations in $\operatorname{Fib}(B)$, the construction of the latter two simplify to a single coidentifier (respectively coinverter) followed by an internal discrete opfibration (resp. fibrewise opfibration in groupoids). We show how these results follow from their analogues in Cat, providing suitable conditions on a 2-category $\mathcal{C}$, that allow the transfer of the construction of coinverters and coidentifiers from $\mathcal{C}$ to $\operatorname{Fib}_{\mathcal{C}}(B)$.
\end{abstract}

Keywords Internal fibration · Factorization system $\cdot$ Coidentifier $\cdot$ Coinverter

Mathematics Subject Classification 18A32 $\cdot 18$ D05 $\cdot 18 D 30$

\section{Introduction}

The crucial point of the work [2] was to recover Yoneda's Classification Theorem in [17, $\S 3.2]$ as a result of the factorization of a regular span $S: X \rightarrow B \times A$ through an internal discrete opfibration, in the 2-category $\operatorname{Fib}(B)$ of fibrations over $B$. Yoneda's Theorem was the base to give a new interpretation of cohomology groups in the additive case. Theorem 3.2

Communicated by Nicola Gambino.

Partial financial support was received from INDAM-Istituto Nazionale di Alta Matematica "Francesco Severi”-Gruppo Nazionale per le Strutture Algebriche, Geometriche e le loro Applicazioni.

Giuseppe Metere

giuseppe.metere@unipa.it

1 Institut de recherche en mathématique et physique, Université catholique de Louvain, Chemin du Cyclotron 2, 1348 Louvain-la-neuve, Belgium

2 Dipartimento di Matematica, Università degli Studi di Milano, Via C. Saldini, 50, 20133 Milan, Italy

3 Dipartimento di Matematica e Informatica, Università degli Studi di Palermo, Via Archirafi 34, 90123 Palermo, Italy 
in [2], which extends the above factorization to any fibrewise opfibration (see Definition 4.7) with codomain a split fibration, allows us to enlarge this point of view to non-additive cases, such as cohomology of groups and of associative algebras.

The first aim of the present work is to show that the above mentioned result, obtained in [2] via an ad hoc construction, is actually an application of an orthogonal factorization system in $\operatorname{Fib}(B)$, whose right class is given by internal discrete opfibrations. This is explained in Theorem 4.10, which generalizes to $\mathrm{Fib}(B)$ the well known comprehensive factorization system of Cat [15]. The above theorem relies on the fact that, for the class of opfibrations, the comprehensive factorization system coincides with the orthogonal factorization system in Cat given by (sequence of coidentifiers, discrete functor), whose construction in this case simplifies to a single coidentifier (see Theorem 4.1 and Corollary 4.2). Actually, a similar phenomenon occurs in $\mathrm{Fib}(B)$. In fact, $\mathrm{Fib}(B)$ inherits from Cat the latter factorization system (see Theorem $4.5(i)$ ), which, restricted to fibrewise opfibrations, can be performed by a single coidentifier. This implies that for such class it coincides with the internal comprehensive factorization system (see Corollary 4.11).

As an obvious consequence of the results reported so far, we get a reflection of any morphism in $\operatorname{Fib}(B)$ onto a discrete morphism. For some purposes, this may cause an undesired loss of information with respect to the initial data. So, one may look for a finer reflection, where fibres are turned into groupoids. This gives a richer structure which is at the base, for example, of the interpretation given in [1] of Schreier-Mac Lane Theorem on the classification of group extension and its further generalizations (see Proposition 2.7 in [1]).

The main goal of the present work is to show first in Theorem $4.5(i i)$ that $\mathrm{Fib}(B)$ inherits such a finer factorization from the one in Cat given by (sequence of coinverters, conservative functor) introduced in [7]; second, that for fibrewise opfibrations, it is performed by a single coinverter followed by a fibrewise opfibration whose fibres are groupoids (Theorem 4.12).

The achieved results rely on specific 2-categorical properties of Cat and of $\operatorname{Fib}(B)$ which are studied in detail in Sect. 3. In particular, in Proposition 3.8 we detect a sufficient condition to transfer the construction of coidentifiers and coinverters from a 2-category $\mathcal{C}$ to the 2category $\operatorname{Fib}_{\mathcal{C}}(B)$ of internal fibrations over a fixed object $B$. This happens when the 2-monad $R: \mathcal{C} / B \rightarrow \mathcal{C} / B$, whose pseudo-algebras define internal fibrations (in the sense of Street [10]), preserves coidentifiers and coinverters of identees. In Propositions 3.13 and 3.16 we prove that the property stated above holds when $\mathcal{C}=$ Cat and $\mathcal{C}=\operatorname{Fib}(B)$, for any $B$, thanks to the exponentiability of fibrations and opfibrations in Cat.

Throughout the paper, 2-limits and 2-colimits are to be understood in a strict sense.

\section{Review of Internal Fibrations}

Let $\mathcal{C}$ be a finitely complete 2-category [11]. For a fixed object $B$ in $\mathcal{C}$, we shall denote by $\mathcal{C} / B$ the slice 2-category over $B$ and by $\mathcal{C} / / B$ the pseudo-slice 2-category over $B$.

We shall denote as follows the (strict) comma objects in $\mathcal{C}$ of identities, along identities on the left and on the right respectively, and iso-comma along identities:
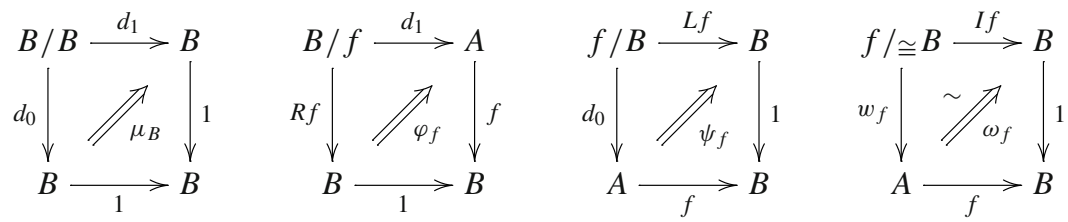
Following [10], one can extend the assignment $f \mapsto L f$ to 1-cells and 2-cells, yielding a 2-functor $L: \mathcal{C} / / B \rightarrow \mathcal{C} / / B$. Moreover there exist: a pseudo 2-natural transformation $u: 1_{\mathcal{C} / / B} \rightarrow L$, a (strict) 2-natural transformation $m: L^{2} \rightarrow L$ and a modification $\lambda: L u \rightarrow$ $u L$ such that $(L, u, m, \lambda)$ gives rise to a KZ-doctrine in the sense of Definition 1.1 in [9]. In other words, this structure provides a lax-idempotent 2-monad.

Let us observe that, in fact, $L: \mathcal{C} / / B \rightarrow \mathcal{C} / / B$ factors through the inclusion of $\mathcal{C} / B$ in $\mathcal{C} / / B$, and the above 2-monad on $\mathcal{C} / / B$ restricts to a strict 2 -monad on $\mathcal{C} / B$, which is also part of a KZ-doctrine by the same $\lambda$. We will adopt the same notation for both monads as far as no confusion arises.

Like $L$, also the 2-functors $R$ and $I$ on $\mathcal{C} / / B$, defined by the corresponding comma squares in (1), can be endowed with a structure of 2-monad, which is colax-idempotent in the case of $R$ and pseudo-idempotent in the case of $I$. In both cases, these structures restrict to strict 2-monads $(R, v, n, \rho)$ and $(I, i, l, \iota)$ on $\mathcal{C} / B$.

One of the most important features of KZ-doctrines is that the corresponding (pseudo-) algebra structures are unique up to isomorphism for each object and they are characterized as right (pseudo-)inverse left adjoint to the unit component of the monad. Applying this observation and its dual to the special cases of the 2-functors $L, R$ and $I$ described above, one can characterize (pseudo-)opfibrations (and dually fibrations) and isofibrations in $\mathcal{C}$.

Proposition 2.1 For a morphism $f: A \rightarrow B$ in $\mathcal{C}$ the following conditions are equivalent and define an internal fibration (respectively pseudo-fibration):

1. (i) For all $X$ in $\mathcal{C}, \mathcal{C}(X, f): \mathcal{C}(X, A) \rightarrow \mathcal{C}(X, B)$ is a fibration (respectively pseudofibration) in Cat;

(ii) for all $g: Y \rightarrow X$, the commutative square below is a morphism of fibrations (respectively pseudo-fibrations) in Cat:

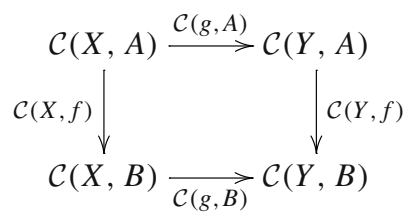

2. $f$ admits a structure of pseudo-algebra for the 2-monad $R: \mathcal{C} / B \rightarrow \mathcal{C} / B$ (respectively $R: \mathcal{C} / / B \rightarrow \mathcal{C} / / B)$

3. The morphism $v_{f}: f \rightarrow R f$ admits a right adjoint in $\mathcal{C} / B$ (respectively $\mathcal{C} / / B$ );

4. (Chevalley criterion) The morphism $f_{1}: A / A \rightarrow B / f$, determined by the equations

$$
\left\{\begin{array}{l}
(R f) f_{1}=f d_{0} \\
d_{1} f_{1}=d_{1} \\
\varphi_{f} f_{1}=f \mu_{A}
\end{array}\right.
$$

admits a right adjoint in $\mathcal{C}$ with counit an identity (respectively isomorphism).

In practice, given an internal fibration according to the above form 2 of Proposition 2.1, it is convenient to fix a corresponding pseudo-algebra structure once and for all (which in Cat means to fix a cleavage). Accordingly, throughout the paper, $\operatorname{Fib}_{\mathcal{C}}(B)$ will denote the 2-category whose objects are pseudo-algebras for the monad $R: \mathcal{C} / B \rightarrow \mathcal{C} / B$, whose 1-cells are strict pseudo-algebra morphisms, and with the obvious 2-cells (we shall write just $\operatorname{Fib}(B)$ for $\mathcal{C}=$ Cat). 
Remark 2.2 The definition of internal fibration (resp. pseudo-fibration) in a representable 2-category appears in the form 2 of Proposition 2.1 in the works of Street [10,12]. The characterizations 1 and 3 in Proposition 2.1 are well-known and already present in the literature (see, for example, [16]). As for the Chevalley criterion, it was first proved by Gray [4] for fibrations in Cat, while an internal version of it appears in [10, Proposition 9], asking for the unit to be an isomorphism. In fact, the latter characterizes pseudo-opfibrations (see (3.17) in [12]). This is the reason why we consider the characterization 4 also for internal (strict) fibrations.

Definition 2.3 An internal fibration is said to be discrete if the functor $\mathcal{C}(X, f)$ in 1. (i) of Proposition 2.1 is a discrete fibration.

The equivalent conditions of Proposition 2.1 may be easily adapted to define internal opfibrations (respectively pseudo-opfibrations), replacing the monad $R$ with the monad $L$. For the reader's convenience, throughout the paper, most of the results are stated in terms of fibrations. Where not explicitly provided, the corresponding results for opfibrations can be obtained by duality.

Proposition 2.4 For a morphism $f: A \rightarrow B$ in $\mathcal{C}$ the following conditions are equivalent and define an internal isofibration:

1. For all $X$ in $\mathcal{C}, \mathcal{C}(X, f): \mathcal{C}(X, A) \rightarrow \mathcal{C}(X, B)$ is an isofibration in Cat;

2. $f$ admits a structure of pseudo-algebra for the 2-monad $I: \mathcal{C} / B \rightarrow \mathcal{C} / B$;

3. the morphism $i_{f}: f \rightarrow$ If admits a right adjoint in $\mathcal{C} / B$.

\section{Coinverters and Coidentifiers in Fib $_{\mathcal{C}}(B)$}

From now on, let $\mathcal{C}$ be a finitely complete 2 -category with coidentifiers and coinverters of reflexive 2-cells, whose definition we recall for the sake of completeness (the reader may refer to $[8,13]$ for example).

Definition 3.1 The coidentifier (respectively coinverter) of a 2-cell $\alpha$ is a 1-cell $q$ such that:

1. $q \alpha$ is an identity (resp. isomorphism);

2. for any other 1-cell $f$ such that $f \alpha$ is an identity (resp. isomorphism), there exists a unique 1-cell $t$ with $t q=f$;

3. for any 2-cell $\beta: f \rightarrow f^{\prime}$ such that $f \alpha$ and $f^{\prime} \alpha$ are identities (resp. isomorphisms), there exists a unique 2-cell $\gamma$ with $\gamma q=\beta$;

In this paper we will consider in particular coidentifiers (coinverters) of identees (invertees). Given a 1-cell $f$, we denote by $(K, \kappa)$ its identee, where $\kappa$ is the 2-universal 2-cell making $f \kappa$ an identity. We denote by $(W, \omega)$ the invertee of $f$, where $\omega$ is the 2-universal 2-cell making $f \omega$ an isomorphism.

Later on, we will take advantage of the following results concerning isofibrations. Recall that a 2 -cell is called $f$-vertical if its image under $f$ is an identity.

Lemma 3.2 Let $f$ be an isofibration and $\alpha$ a 2-cell such that $f \alpha$ is an isomorphism. Then $\alpha$ factorizes as $\alpha=\sigma \cdot \tau$, where $\tau$ is $f$-vertical and $\sigma$ is an isomorphism.

Proof Since $f$ is an isofibration, the isomorphism $f \alpha$ admits a cartesian lifting $\sigma$, which is an isomorphism, at the codomain of $\alpha$. Then $\tau$ is the unique $f$-vertical factorization of $\alpha$ through $\sigma$. 
Corollary 3.3 Let $f$ be an isofibration, then:

(i) $f$ is conservative if and only if its fibres are groupoids;

(ii) the coinverter of the identee of $f$ coincides with the coinverter of its invertee.

Proof Let $(W, \omega)$ and $(K, \omega c)$ be the invertee and the identee, respectively, of $f$ :

$$
K \stackrel{c}{\longrightarrow} W \overbrace{\frac{w_{0}}{w_{1}}}^{\downarrow} A \stackrel{f}{\longrightarrow} B .
$$

Since $f \omega$ is an isomorphism by definition, as in Lemma 3.2, we can factorize $\omega$ as a composite $\omega=\sigma \cdot \tau$, where $\sigma$ is a cartesian lifting of $f \omega$, and $\tau$ the unique $f$-vertical comparison 2-cell. $\tau$ being vertical, there is a unique $c^{\prime}: W \rightarrow K$ such that $\omega c c^{\prime}=\tau$. So we have factorized $\omega$ as in the following diagram:

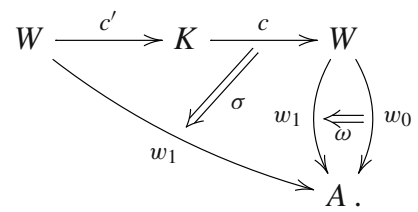

It is now easy to see that $f$ is conservative, i.e. its invertee is an isomorphism, if and only if its fibres are groupoids, i.e. its identee is an isomorphism.

As for the second statement, it suffices to observe that the coinverter of the identee $\omega c$ coinverts also $\omega=\sigma \cdot \omega c c^{\prime}$.

Obviously the last result does not hold in general if $f$ is not an isofibration, as it is witnessed by the non-constant functor from the arrow-category 2 to the groupoid I with two objects and two non-trivial arrows.

Identees in $\mathcal{C} / B$ are computed in $\mathcal{C}$, while this is not true for invertees (and 2-limits in general). On the other hand, it is easy to check that the following holds for 2-colimits.

Lemma 3.4 The forgetful 2-functor dom: $\mathcal{C} / B \rightarrow \mathcal{C}$ creates 2-colimits, and in particular coidentifiers and coinverters of identees.

We are going to explore the behaviour of the monad $R$ with respect to these limits and colimits. Analogous results can be proved for the monad $L$.

Remark 3.5 It is worth observing that the functor $R$ can be described by means of the following construction:

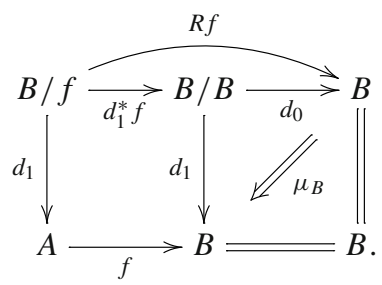

That is, $R=\left(d_{0}\right) ! d_{1}^{*}$, i.e. the composite of the change-of-base 2-functor along $d_{1}$ with the composition 2-functor with $d_{0}$, which is left adjoint to $d_{0}^{*}$. 
Lemma 3.6 The monad $R: \mathcal{C} / B \rightarrow \mathcal{C} / B$ preserves identees.

Proof By Remark 3.5, the thesis follows from the fact that $d_{1}^{*}$ preserves limits, being a right adjoint, and $\left(d_{0}\right)$ ! preserves identees.

Lemma 3.7 The identee of a morphism $p:(A, f) \rightarrow(C, g)$ in $\mathrm{Fib}_{\mathcal{C}}(B)$ can be computed as in $\mathcal{C}$.

\section{Proof Let}

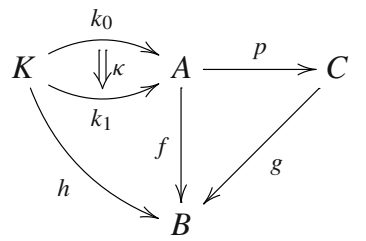

be an identee diagram in $\mathcal{C} / B$ (which means that $\kappa$ is also the identee of $p$ in $\mathcal{C}$ ). Since $p$ is a morphism in $\operatorname{Fib}_{\mathcal{C}}(B)$ and $R$ preserves identees by Lemma 3.6, it is straightforward to prove that the adjunctions $v_{f} \dashv r_{f}$ and $v_{g} \dashv r_{g}$ in the diagram

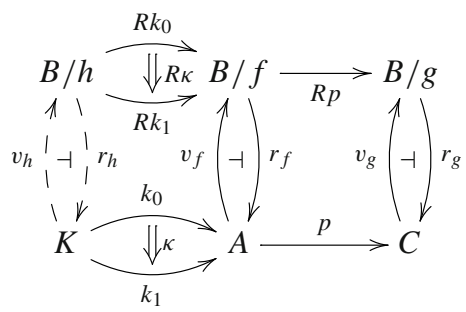

induce an adjunction $v_{h} \dashv r_{h}$ by the universal property of the identees.

There's no obvious reason why coinverters and coidentifiers should be preserved by the monad $R$, however this happens in some cases of interest which we will explore later on. So, for a given object $B$ in $\mathcal{C}$, we shall consider the property

$(\dagger)$ The monad $R: \mathcal{C} / B \rightarrow \mathcal{C} / B$ preserves coidentifiers and coinverters of identees.

Proposition 3.8 Let $B$ be an object in $\mathcal{C}$ satisfying $(\dagger), p:(A, f) \rightarrow(C, g)$ a morphism in $\operatorname{Fib}_{\mathcal{C}}(B)$ and $\kappa$ its identee in $\mathcal{C}$. Then we get a factorization

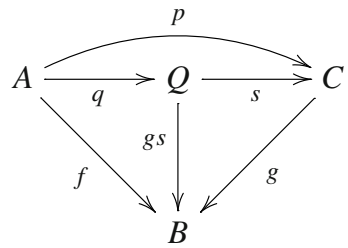

of $p$ in $\mathrm{Fib}_{\mathcal{C}}(B)$ in either of the following ways:

(i) taking as $q$ the coidentifier of $\kappa$ in $\mathcal{C}$; then $q:(A, f) \rightarrow(Q, g s)$ is the coidentifier of $\kappa$ in $\operatorname{Fib}_{\mathcal{C}}(B)$;

(ii) taking as $q$ the coinverter of $\kappa$ in $\mathcal{C}$; then $q:(A, f) \rightarrow(Q, g s)$ is the coinverter of $\kappa$ in $\operatorname{Fib}_{\mathcal{C}}(B)$. 
Proof We shall only prove $(i i),(i)$ is proved analogously.

Recall that $\kappa$ is also the identee of $p$ in $\mathcal{C} / B$ and consider the corresponding identee diagram (2) in $\mathcal{C} / B$. Since $p$ (and then $f$ ) coinverts $\kappa$, the morphisms $s$ and $g s$ in the factorization above are uniquely determined by the universal property of $q$, and this explains why $q$ is a coinverter of $\kappa$ in $\mathcal{C} / B$.

Since $f$ is a fibration, the unit component $v_{f}:(A, f) \rightarrow(B / f, R f)$ admits a right adjoint $r_{f}$ in $\mathcal{C} / B$. We call $\eta_{f}$ and $\epsilon_{f}$ the corresponding unit and counit. Likewise, $v_{g}$ has a right adjoint $r_{g}$, and $p r_{f}=r_{g}(R p)$ since $p$ is a morphism in $\operatorname{Fib}_{\mathcal{C}}(B)$. Let us consider the following diagram:

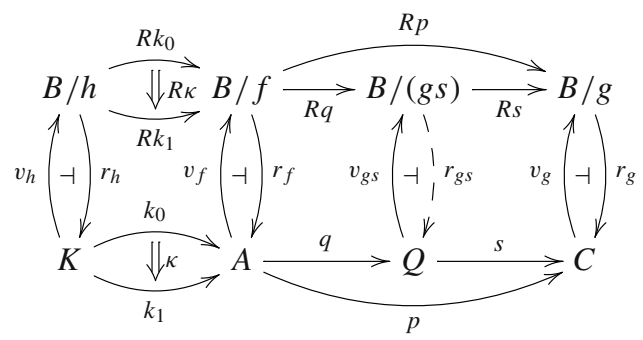

Now, $p r_{f}(R \kappa)=r_{g}(R p)(R \kappa)=1$, hence $r_{f}(R \kappa)$ factors through $\kappa$ and $q r_{f}(R \kappa)$ is an isomorphism. By the assumption ( $\dagger), R q$ is the coinverter of $R \kappa$, so there exists a unique $r_{g s}: B /(g s) \rightarrow Q$ such that $r_{g s}(R q)=q r_{f}$. By the 2-dimensional universal property of the coinverters $q$ and $R q$, one can prove that a unit $\eta_{g s}$ and a counit $\epsilon_{g s}$ are induced by $\eta_{f}$ and $\epsilon_{f}$ respectively, making $v_{g s} \dashv r_{g s}$ an adjoint pair in $\mathcal{C} / B$, so that $g s$ is a fibration. As a consequence of this construction, $q$ turns out to be a morphism of fibrations over $B$.

It remains to show that for each $c:(A, f) \rightarrow(Y, y)$ in $\operatorname{Fib}_{\mathcal{C}}(B)$ such that $c \kappa$ is an isomorphism, the unique comparison morphism $t$ in $\mathcal{C} / B$, induced by the coinverter $q$ and such that $t q=c$, is actually a morphism in $\operatorname{Fib}_{\mathcal{C}}(B)$. Let us denote by $r_{y}$ the $R$-pseudo-algebra structure on $y$, i.e. the right adjoint to $v_{y}$, and observe that the diagram

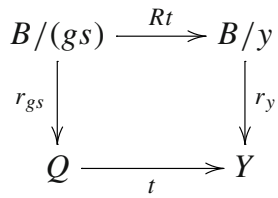

commutes since $R q$ is a coinverter, then epimorphic, and precomposition with $R q$ gives the commutative square presenting $c$ as a morphism of $R$-pseudo-algebras.

Corollary 3.9 Let $B$ be an object in $\mathcal{C}$ satisfying $(\dagger), f: A \rightarrow B$ a fibration in $\mathcal{C}, \kappa$ its identee in $\mathcal{C}$, and $q: A \rightarrow Q$ its coinverter (respectively coidentifier) in $\mathcal{C}$. Then the unique comparison morphism $s: Q \rightarrow B$, such that $s q=f$, is a fibration and $q$ is the coinverter (respectively coidentifier) of $\kappa$ in $\operatorname{Fib}_{\mathcal{C}}(B)$.

Proof Apply Proposition 3.8 to the morphism $f:(A, f) \rightarrow\left(B, 1_{B}\right)$ in $\operatorname{Fib}_{\mathcal{C}}(B)$.

In the cases we are interested in, which will be studied in Sect. 3.1, the property $(\dagger)$ relies upon the exponentiability of split opfibrations in $\mathcal{C}$. In this context, exponentiability is to be understood in a 2-categorical sense: a 1-cell $f$ is exponentiable if the change-of-base 2-functor along $f$ has a right adjoint. 
Lemma 3.10 If for an object $B$ in $\mathcal{C}$, the comma projection $d_{1}$ in the diagram

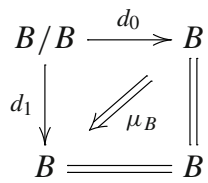

is exponentiable, then the functor

$$
R: \mathcal{C} / B \rightarrow \mathcal{C} / B
$$

has a right adjoint, hence $B$ satisfies the condition ( $\dagger)$. In particular, this holds for any $B$ when split opfibrations in $\mathcal{C}$ are exponentiable.

Proof By Remark 3.5, $R=\left(d_{0}\right) ! d_{1}^{*}$. Hence $R$ is left adjoint to $\left(d_{1}\right)_{*} d_{0}^{*}$, where $\left(d_{1}\right)_{*}$ denotes the right adjoint to $d_{1}^{*}$, which exists by assumption.

Proposition 3.11 Under the assumptions of Lemma 3.10, the functor $\mathrm{dom:} \operatorname{Fib}_{\mathcal{C}}(B) \rightarrow \mathcal{C}$ creates 2-colimits.

Proof One can repeat the same arguments of the proof of Proposition 3.8, using the fact that now $R$ preserves any 2 -colimit, and that dom: $\mathcal{C} / B \rightarrow \mathcal{C}$ creates 2 -colimits.

Remark 3.12 If instead of $(\dagger)$ we ask for

$\left(\dagger^{\prime}\right)$ The monad $L: \mathcal{C} / B \rightarrow \mathcal{C} / B$ preserves coidentifiers and coinverters of identees, then the results of Proposition 3.8 hold with $\operatorname{Fib}_{\mathcal{C}}(B)$ replaced by $\operatorname{OpFib}_{\mathcal{C}}(B)$. Accordingly, if $d_{0}$ is exponentiable, and in particular when split fibrations are exponentiable in $\mathcal{C}$, then $L$ admits a right adjoint, $\left(\dagger^{\prime}\right)$ holds for $B$, and dom: $\operatorname{OpFib}_{\mathcal{C}}(B) \rightarrow \mathcal{C}$ creates colimits.

\subsection{Case Study: Cat and Fib $(B)$}

It is well-known that fibrations in Cat are exponentiable [3] in the classical 1-categorical sense. As observed by Johnstone [6], this property holds also in the 2-categorical sense recalled above. As a consequence, by Lemma 3.10 and Remark 3.12, we have:

Proposition 3.13 In the 2-category Cat, each object $B$ satisfies the conditions $(\dagger)$ and $\left(\dagger^{\prime}\right)$.

We will see in Proposition 3.16 that one can extend the last property from Cat to $\operatorname{Fib}(B)$ for each $B$, by means of the pseudo-functorial interpretation of fibrations in Cat.

Proposition 3.14 Let $B$ be a category. Then

(i) 2-colimits, in particular coidentifiers and coinverters of identees, exist in $\mathrm{Fib}(B)$;

(ii) their construction can be performed fibrewise.

Proof ( $i$ ) The existence is guaranteed by Proposition 3.11, since Cat is 2-cocomplete. We shall prove $(i i)$ just for coidentifiers, the general case can be treated likewise.

Let us consider a morphism $p:(A, f) \rightarrow(C, g)$ in $\operatorname{Fib}(B)$ and focus our attention on its restriction $p_{b}$ to a single fibre over some $b$ in $B$. We can consider the coidentifier $q_{b}: A_{b} \rightarrow Q_{b}$ of its identee $\left(K_{b}, \kappa_{b}\right)$. Since $f$ and $g$ are fibrations, the assignments $b \mapsto A_{b}$ and $b \mapsto C_{b}$ are pseudo-functorial and the collection of the functors $p_{b}$ gives rise to a 
natural transformation of pseudo-functors from $B^{o p}$ to Cat. By the universal property of the coidentifiers $q_{b}$ for each $b$, the assignment $b \mapsto Q_{b}$ is also pseudo-functorial and the $q_{b}$ 's organize in a natural transformation. Let us briefly show how this can be proved.

In fact, the assignment $b \mapsto K_{b}$ is also pseudo-functorial and together with the collection of the $\kappa_{b}$ 's, it determines the identee $(K, \kappa)$ of the cartesian functor $p$. Given an arrow $\beta: b^{\prime} \rightarrow b$ in $B$, we always denote by $\beta^{*}$ its associated change of base functor for any chosen fibration over $B$. Since $q_{b^{\prime}} \beta^{*} \kappa_{b}=q_{b^{\prime}} \kappa_{b^{\prime}} \beta^{*}=1$, by the universal property of the coidentifier $q_{b}$ there is a unique functor $\beta^{*}: Q_{b} \rightarrow Q_{b}^{\prime}$ such that $\beta^{*} q_{b}=q_{b^{\prime}} \beta^{*}$ :

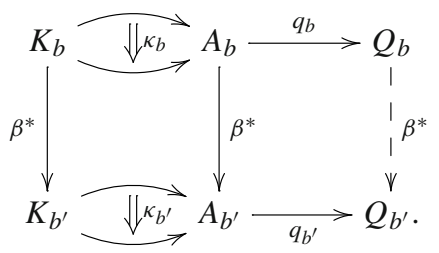

Given a composable pair of arrows

$$
b^{\prime \prime} \stackrel{\beta^{\prime}}{\longrightarrow} b^{\prime} \stackrel{\beta}{\longrightarrow} b
$$

in $B$, let $\phi_{\beta, \beta^{\prime}}:\left(\beta^{\prime}\right)^{*} \beta^{*} \rightarrow\left(\beta \beta^{\prime}\right)^{*}$ be the corresponding coherence isomorphism induced by the fibration $f$. Since $q_{b^{\prime \prime}} \phi_{\beta, \beta^{\prime}}$ is a 2-cell between $q_{b^{\prime \prime}}\left(\beta^{\prime}\right)^{*} \beta^{*}=\left(\beta^{\prime}\right)^{*} \beta^{*} q_{b}$ and $q_{b^{\prime \prime}}\left(\beta \beta^{\prime}\right)^{*}=$ $\left(\beta \beta^{\prime}\right)^{*} q_{b}$, then by the universal property of the coidentifier $q_{b}$ there exists a unique invertible 2-cell $\psi_{\beta, \beta^{\prime}}$ such that $\psi_{\beta, \beta^{\prime}} q_{b}=q_{b^{\prime \prime}} \phi_{\beta, \beta^{\prime}}$.

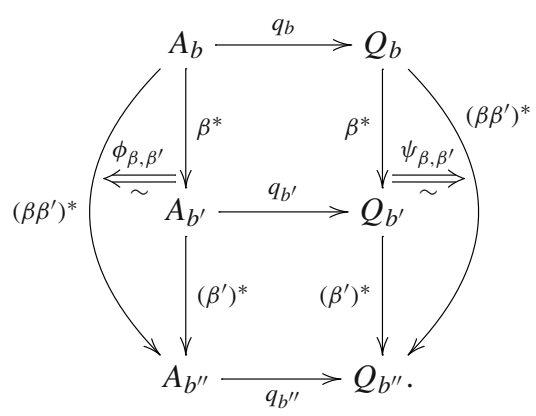

Finally, the coherence conditions on the $\psi$ 's making the assignment $b \mapsto Q_{b}$ into a pseudofunctor can be deduced once again by the universal property of the $q_{b}$ 's. Since for a morphism $t:(A, f) \rightarrow(Y, y)$ in $\operatorname{Fib}(B), t \kappa=1$ if and only if $t_{b} \kappa_{b}=1$ for each $b$ in $B, q$ is actually the coidentifier of $\kappa$ in $\operatorname{Fib}(B)$.

Lemma 3.15 For each internal fibration (resp. opfibration) $p:(E, e) \rightarrow(A, a)$ in $\operatorname{Fib}(B)$, the change of base 2-functor $p^{*}: \mathrm{Fib}(B) /(A, a) \rightarrow \mathrm{Fib}(B) /(E, e)$ preserves coidentifiers and coinverters of identees.

Proof We will prove the result concerning internal fibrations and coinverters, the variations involving opfibrations and coidentifiers are obtained analogously. 
Let the arrow $q:((C, c), f) \rightarrow((D, d), g)$ in the diagram

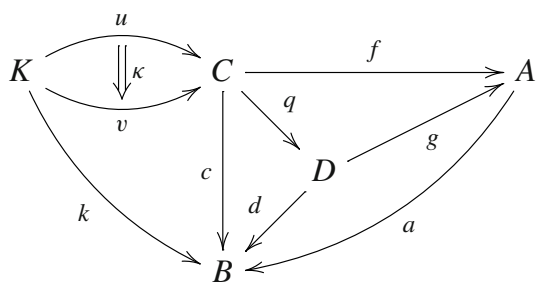

be the coinverter in $\mathrm{Fib}(B) /(A, a)$ of an identee $\kappa$, and consider its image under the change of base 2-functor $p^{*}$, i.e. the upper part of the next diagram (we omit all arrows over $B$, all pullbacks provide in fact fibrations over $B$ ):

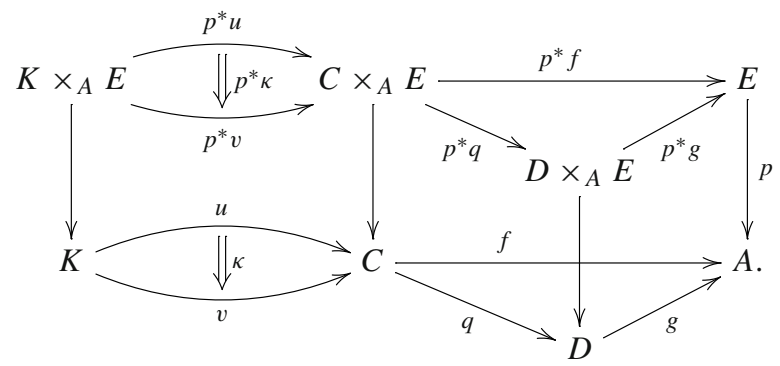

We would like to show that $p^{*} q$ is the coinverter of the identee $p^{*} \kappa$ in $\operatorname{Fib}(B) /(E, e)$. To this end, we consider the restriction of the above diagram to the fibres over any object $b$ in $B$. By limit commutation, the latter is the same as the corresponding change of base diagram in the fibres over $b$ :

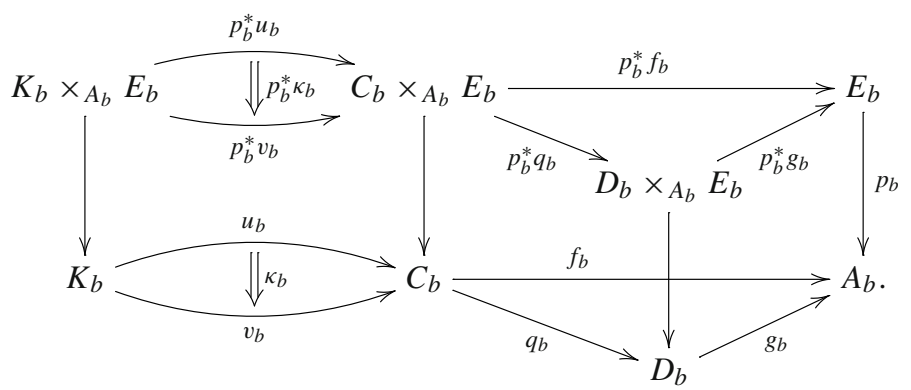

Now observe that, by Proposition 3.14 (ii), $q_{b}$ is the coinverter of $\kappa_{b}$. Moreover, since $p_{b}$ is a fibration in Cat by assumption, it is exponentiable, hence $p_{b}^{*}$ is a left adjoint and $p_{b}^{*} q_{b}$ is the coinverter of $p_{b}^{*} \kappa_{b}$. Finally, again by Proposition $3.14, p^{*} q$ is the coinverter of $p^{*} \kappa$ in $\operatorname{Fib}(B)$, and hence in $\operatorname{Fib}(B) /(A, a)$.

Proposition 3.16 In the 2-category $\mathrm{Fib}(B)$, each object satisfies the conditions $(\dagger)$ and $\left(\dagger^{\prime}\right)$. 
Proof Let $a: A \rightarrow B$ be a fibration of categories, then the projections $d_{0}$ and $d_{1}$ of the comma square in $\operatorname{Fib}(B)$

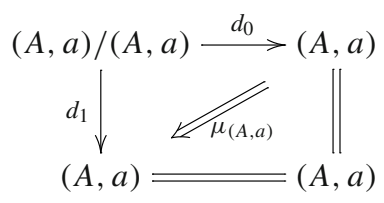

are an internal fibration and opfibration respectively (see Theorem 14 in [10]). As a consequence, by Lemma 3.15, the corresponding change of base 2-functors $d_{0}^{*}$ and $d_{1}^{*}$ preserve coidentifiers and coinverters of identees. Now likewise in the proof of Lemma 3.10, the thesis follows from the fact that $R=\left(d_{0}\right) ! d_{1}^{*}$ and $\left(d_{0}\right)$ ! is a left adjoint (and similarly for $L$ ).

Proposition 3.17 Let $p:(A, f) \rightarrow(C, g)$ be an internal fibration (resp. opfibration) in $\mathrm{Fib}(B)$. Then the morphism $s$ in the factorization of Proposition $3.8(i)$ is an internal fibration (resp. opfibration) in $\mathrm{Fib}(B)$.

Proof By Proposition 3.16, we can apply Corollary 3.9 to the fibration $p$ in $\operatorname{Fib}(B)$.

\section{Three Factorization Systems in Fib(B)}

Let us recall that an (orthogonal) factorization system on a 1-category $\mathcal{C}$ is given by a pair $(\mathcal{E}, \mathcal{M})$ of classes of morphisms in $\mathcal{C}$ such that:

(i) $\mathcal{E}$ and $\mathcal{M}$ are closed under composition and contain isomorphisms;

(ii) each morphism $f$ in $\mathcal{C}$ admits a factorization $f=m \cdot e$ with $m$ in $\mathcal{M}$ and $e$ in $\mathcal{E}$;

(iii) for each commutative square

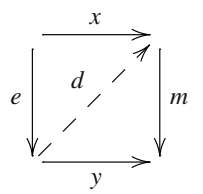

with $m$ in $\mathcal{M}$ and $e$ in $\mathcal{E}$, there exists a unique morphism $d$ such that $m d=y$ and $d e=x$.

When $\mathcal{C}$ is a 2-category such factorization $\operatorname{system}(\mathcal{E}, \mathcal{M})$ becomes a strict 2-categorical (or $a$ Cat-enriched) factorization system if the following additional property holds:

(iv) for each diagram

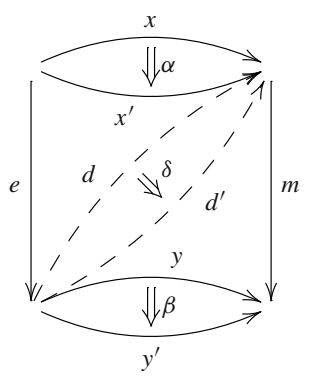


where $m \alpha=\beta e$ and $d$ and $d^{\prime}$ are determined by $(x, y)$ and $\left(x^{\prime}, y^{\prime}\right)$ respectively, there exists a unique $\delta$ such that $m \delta=\beta$ and $\delta e=\alpha$.

\subsection{Three Factorization Systems in Cat}

The comprehensive factorization system introduced in [15] and given by (initial functor, discrete opfibration) is a well known example of factorization system in Cat, which is actually strict 2-categorical. We will call by the same name the dual factorization given by (final functor, discrete fibration).

Another example of factorization system in Cat, which is 2-categorical as well, and has conservative functors as right class, was introduced in [7, Theorem C.0.31] relying on the notion of coinverter. Let us illustrate how this is obtained. For a functor $f$

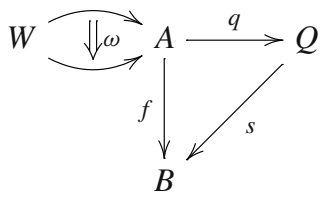

the factorization $s$ of $f$ through the coinverter $q$ of the invertee $\omega$ of $f$ is not conservative in general. One has to repeat this "invertee-coinverter" procedure possibly infinitely many times in order to get a conservative comparison, and an actual factorization system. A similar phenomenon occurs when taking the "identee-coidentifier" analogue of the previous procedure, which allows to factor any functor as a (possibly infinite) sequence of coidentifiers followed by a discrete functor, i.e. a functor whose fibres are discrete, yielding another (strict 2-categorical) factorization system in Cat.

We are going to show that if we restrict ourselves to fibrations, the latter two transfinite procedures above reduce to a single step. We start with the second one and we show also that, for fibrations, it coincides with the comprehensive factorization system. This actually holds not only in Cat, but in any 2-category $\operatorname{Cat}(\mathcal{E})$ of internal categories where the construction of the comprehensive factorization of any functor provided in [14] is still valid, as, for example, when $\mathcal{E}$ is a finitely cocomplete locally cartesian closed category, like any topos $\mathcal{E}$.

Theorem 4.1 Let $f: A \rightarrow B$ be a fibration in $\operatorname{Cat}(\mathcal{E})$ as above. The coidentifier of the identee of $f$ factorizes $f$ into a final functor followed by a discrete fibration, giving then the comprehensive factorization of $f$. Starting with $f$ opfibration, the same procedure yields the dual factorization of $f$ given by an initial functor followed by a discrete opfibration.

Proof We consider just the case of fibrations. Following the approach of Section 3 in [14], we perform the comprehensive factorization of $f$ by taking the free $R$-algebra $R f$, which is a split fibration, and then reflecting it into a discrete fibration $p$ : 


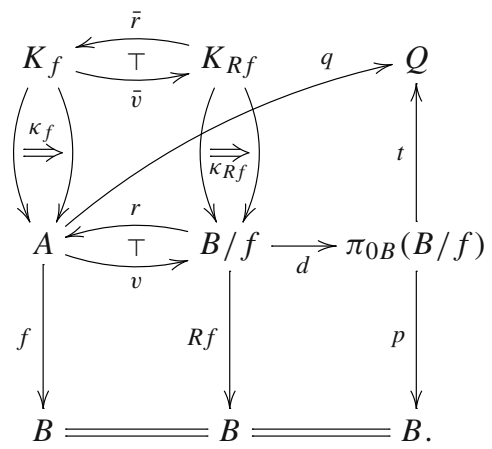

By construction of the above reflection, $d$ is the coidentifier of the identee $\kappa_{R f}$ of $R f$. Considering the adjunction $v \dashv r$ provided by the fact that $f$ is a fibration, we get $d v \kappa_{f}=$ $d \kappa_{R f} \bar{v}=1$, where $\kappa_{f}$ is the identee of $f$. Let now $q$ be a functor such that $q \kappa_{f}=1$, and consider the unit $\eta: 1 \rightarrow r v$ of the adjunction $v \dashv r$ in $\operatorname{Cat}(\mathcal{E}) / B$. Then $f \eta=1$ and $\eta$ is contained in $\kappa_{f}$, so that $q \eta=1$ as well, and $q r v=q$. On the other hand, the counit $\epsilon: v r \rightarrow 1$ is such that $(R f) \epsilon=1$, so it is contained in $\kappa_{R f}$ and hence $d \epsilon=1$ and $d v r=d$.

Now, $q r \kappa_{R f}=q \kappa_{f} \bar{r}=1$, so by the universal property of the coidentifier $d$ there exists a unique $t$ such that $t d=q r$. Hence $q=q r v=t d v$, and $t$ is unique with this last property. Indeed, if $t^{\prime} d v=q$ for some $t^{\prime}$, then $t^{\prime} d=t^{\prime} d v r=q r=t d$ and hence $t^{\prime}=t$ since $d$ is epimorphic. This proves that $d v$ is the coidentifier of $\kappa_{f}$, and then it is final [14].

Corollary 4.2 For a fibration (resp. opfibration) $f$, the factorization of $f$ given by (sequence of coidentifiers, discrete functor) reduces to a single coidentifier and coincides with the comprehensive factorization.

In the special case of Cat, Theorem 4.1 can be proved directly by means of the pseudofunctorial interpretation of fibrations. This indeed is what we are going to do in order to obtain the analogous result, where coidentifiers are replaced by coinverters and discrete fibrations are replaced by fibrations in groupoids (i.e. fibrations whose identee is an isomorphism).

Theorem 4.3 Each fibration (respectively opfibration) $f: A \rightarrow B$ in Cat admits a factorization given by the coinverter of the identee of $f$ followed by a fibration (resp. opfibration) in groupoids. This factorization of $f$ coincides with the one given by (sequence of coinverters, conservative functor).

Proof Let us denote by

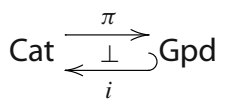

the reflection of categories in groupoids, where the left adjoint $\pi$ can be obtained by taking as unit component, for each category $A$, the coinverter $\eta_{A}$ of the 2-cell $\mu_{A}$ associated with the comma category $A / A$ :

$$
A / A \underset{\Downarrow \mu_{A}}{\longrightarrow} A \stackrel{\eta_{A}}{\longrightarrow} \pi(A) .
$$

Consider now a fibration $f: A \rightarrow B$ and denote by $[f]: B^{\text {op }} \rightarrow$ Cat the corresponding pseudo-functor. The composite $\pi[f]: B^{\text {op }} \rightarrow$ Gpd gives rise to a fibration in groupoids 
$\bar{f}: \bar{A} \rightarrow B$. On the other hand, $\eta[f]$ corresponds to a morphism $q:(A, f) \rightarrow(\bar{A}, \bar{f})$ in $\operatorname{Fib}(B)$ :

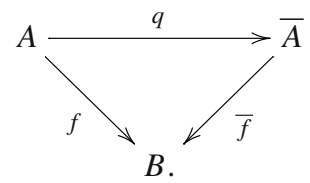

The component $q_{b}$ of $\eta[f]$ at an object $b$ of $B$ is actually the coinverter $\eta_{A_{b}}$ of $\mu_{A_{b}}$ :

$$
A_{b} / A_{b} \stackrel{\Downarrow \mu_{A_{b}}}{\longrightarrow} A_{b} \stackrel{\eta_{A_{b}}}{\longrightarrow} \pi\left(A_{b}\right) .
$$

It is not difficult to see that the pair $\left(A_{b} / A_{b}, \mu_{A_{b}}\right)$ coincides with the restriction $\left(K_{b}, \kappa_{b}\right)$ of the identee $(K, \kappa)$ of $f$ to the fibre over $b$. Hence, as explained in Proposition 3.14, $q$ turns out to be the coinverter of $\kappa$ in $\operatorname{Fib}(B)$. Thanks to Corollary $3.3, \bar{f}$ is conservative and we get the desired factorization of $f$.

\subsection{From Cat to $\operatorname{Fib}(B)$}

We are going to use now the results of the previous sections to produce analogous factorization systems in $\operatorname{Fib}(B)$. First, we need a preliminary result.

Lemma 4.4 For a morphism $p:(A, f) \rightarrow(C, g)$ in $\mathrm{Fib}(B)$, the coinverter $q$ of the invertee of $p$ in $\mathrm{Fib}(B)$ is also the coinverter of the invertee of $p$ in Cat.

Proof The only non-trivial property to prove is that for an arrow $\alpha$ in $A, q \alpha$ is an isomorphism as soon as $p \alpha$ is an isomorphism. But if $p \alpha$ is an isomorphism, then $\alpha$ factors as $\alpha=\kappa \cdot v$ where $\kappa$ is an $f$-cartesian lifting of $f \alpha$, hence an isomorphism, and $v$ is $f$-vertical with $p v$ isomorphism. So $v$, and hence $\alpha$, is inverted by $q$.

Theorem 4.5 For each category $B$, $\mathrm{Fib}(B)$ inherits from Cat two factorization systems given by

(i) (sequence of coidentifiers, discrete cartesian functors);

(ii) (sequence of coinverters, conservative cartesian functors).

Proof Identees in $\operatorname{Fib}(B)$ are computed in Cat by Lemma 3.7. Even if invertees in $\operatorname{Fib}(B)$ may differ from the corresponding invertees computed in Cat, their coinverters coincide by Lemma 4.4. As a consequence, thanks to Proposition 3.11, such factorization systems are just performed in Cat.

Thanks to a result due to Bénabou, proving that a morphism in $\operatorname{Fib}(B)$ is an internal fibration if and only if it is a fibration in Cat (see, for example, Theorem 4.16 in [5]), we easily get the following result.

Proposition 4.6 $\mathrm{Fib}(B)$ inherits from Cat the comprehensive factorization system having internal discrete fibrations as right class.

Proof Let $p:(A, f) \rightarrow(C, g)$ be any morphism in $\operatorname{Fib}(B)$. Take its factorization $(q, s)$ in Cat, with $q$ a final functor and $s$ a discrete fibration. Then $g s$ is a fibration and $s$ is an internal fibration. It is easy to see that, since $p$ is cartesian and $s$ is discrete, $q$ is cartesian as well. 
Our next goal is to show that $\operatorname{Fib}(B)$ admits also a comprehensive factorization system having internal discrete opfibrations as right class. We cannot repeat the above argument because internal opfibrations in $\operatorname{Fib}(B)$ are not opfibrations in Cat. Let us recall from [2] the following definition.

Definition 4.7 (see [2, Definition 2.1]) We say that a morphism $p:(A, f) \rightarrow(C, g)$ in $\operatorname{Fib}(B)$ is a fibrewise (discrete) opfibration if, for every object $b$ of $B$, the restriction $p_{b}: A_{b} \rightarrow C_{b}$ of $p$ to the $b$-fibres is a (discrete) opfibration.

From Theorem 2.8 in [2] it follows that every internal opfibration in $\operatorname{Fib}(B)$ is a fibrewise opfibration, while the latter is exactly a morphism in $\mathrm{Fib}(B)$ which is an internal opfibration in Cat/ $B$ (see Propositions 2.5 and 2.7 in [2]). By Corollary 2.9 in [2], the two notions coincide in the discrete case. Recall also from [2] that Yoneda's regular spans and two-sided fibrations are instances, respectively, of fibrewise opfibrations and internal opfibrations in $\operatorname{Fib}(B)$.

Proposition 4.8 Let $p:(A, f) \rightarrow(C, g)$ be a fibrewise (resp. internal) opfibration in $\mathrm{Fib}(B)$. Then we get a factorization

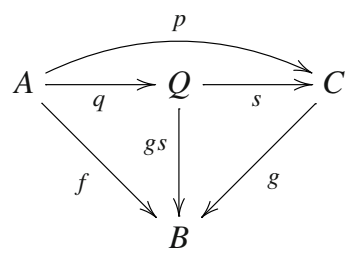

of $p$ in $\mathrm{Fib}(B)$ in either of the following ways:

(i) taking as $q$ the coidentifier of the identee of $p$; then $s$ is a discrete opfibration in $\operatorname{Fib}(B)$;

(ii) taking as $q$ the coinverter of the identee of $p$; then $s$ is a fibrewise (resp. internal) opfibration in groupoids in $\mathrm{Fib}(B)$.

Proof Let us consider a fibrewise opfibration $p:(A, f) \rightarrow(C, g)$ in $\operatorname{Fib}(B)$.

(i) Take the factorization $(q, s)$ of $p$ as in Proposition $3.8(i)$, with $q$ the coidentifier of the identee of $p$. If we restrict to a single fibre over some $b$ in $B$, we get a factorization

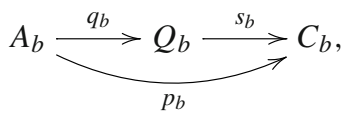

where, thanks to Proposition $3.14(i i), q_{b}$ is the coidentifier of the identee of $p_{b}$. Since the latter is an opfibration, by Theorem $4.1 s_{b}$ is a discrete opfibration, hence $s$ is an internal discrete opfibration in $\mathrm{Fib}(B)$.

(ii) Take instead the factorization $(q, s)$ of $p$ as in Proposition 3.8 (ii). Likewise in (i), for each $b$ in $B$, thanks to Theorem 4.3 , we get a factorization $\left(q_{b}, s_{b}\right)$ of $p_{b}$ into a coinverter followed by an opfibration in groupoids.

If moreover $p$ is an internal opfibration, then $s$ is also an internal opfibration by Corollary 3.9 applied to $\mathrm{Fib}(B)$, thanks to Proposition 3.16 .

The result in Proposition 4.8 ( $i$ ) (with $g$ a split fibration) was obtained in Section 3.3 of [2], by providing an explicit construction of the discrete opfibration $s$ together with an ad hoc definition of $q$, which was later on proved to be the coidentifier of the identee of $p$.

As a consequence of Proposition $4.8(i)$, we get an internal comprehensive factorization system for $\mathrm{Fib}(B)$. In the next results, by initial we mean a morphism which is left orthogonal 
to internal discrete opfibrations. First we need the following lemma, whose dual version is in Proposition 3.5 in [14], for a particular case. For completeness, we formulate it here in a general finitely complete 2-category.

Lemma 4.9 In a finitely complete 2-category, left adjoints are initial.

Proof Consider the commutative square

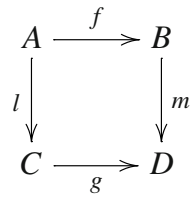

where $m$ is an internal discrete opfibration and $l$ is left adjoint to some $r$, with unit $\eta$ and counit $\epsilon$, so that $\epsilon l \cdot l \eta=1_{l}$ and $r \epsilon \cdot \eta r=1_{r}$.

Let $\gamma: f r \rightarrow d$ denote the opcartesian $m$-lifting of $g \epsilon$ at $f r$, so that $m d=g$. The 2-cell $d \epsilon$ being also an $m$-lifting of $g \epsilon$, it coincides with $\gamma$ since $m$ is a discrete opfibration. Then $m(d \epsilon l \cdot f \eta)=g \epsilon l \cdot m f \eta=g(\epsilon l \cdot l \eta)=1_{g l}$.

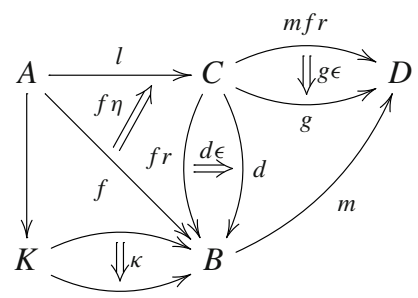

Hence $d \epsilon l \cdot f \eta$ factors through the identee $\kappa$ of $m$, which is discrete, so $\kappa$, and hence $d \epsilon l \cdot f \eta$, is an identity. Consequently, $d l=f$.

Suppose $d^{\prime}: C \rightarrow B$ is another morphism such that $m d^{\prime}=g$ and $d^{\prime} l=f$. Then $d^{\prime} \epsilon$ is another $m$-lifting of $g \epsilon$, so $d^{\prime} \epsilon=d \epsilon$ and $d=d^{\prime}$.

Theorem 4.10 In $\mathrm{Fib}(B)$ there exists a comprehensive factorization system given by (initial morphism, internal discrete opfibration).

Proof Consider a morphism $p:(A, f) \rightarrow(C, g)$ in $\operatorname{Fib}(B)$, and the monad $L: \operatorname{Fib}(B) /(C, g) \rightarrow \operatorname{Fib}(B) /(C, g)$. $L p$ is an internal opfibration, so we can factorize it in $\operatorname{Fib}(B)$ as a coidentifier $q$ followed by an internal discrete opfibration $s$, thanks to Proposition 4.8. Now consider the factorization

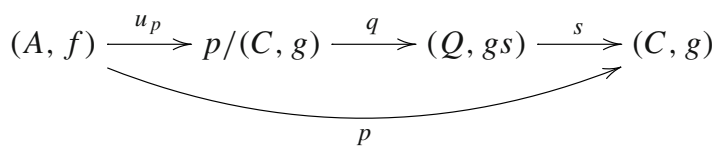

of $p$, where the unit component $u_{p}$ is initial by Lemma 4.9, since it is a left adjoint (see Corollary 6 in [10]). The result follows from Proposition 4.8 as it is easy to see that coidentifiers are initial.

Corollary 4.11 For each fibrewise opfibration, the factorization of Theorem 4.10 coincides with the one given by (sequence of coidentifiers, discrete cartesian functor). 
As a consequence of Proposition 4.8 (ii), we get an extension of Theorem 4.3.

Theorem 4.12 For every fibrewise opfibration $p:(A, f) \rightarrow(C, g)$ in $\operatorname{Fib}(B)$, the factorization of Proposition 4.8 (ii) coincides with the one given by (sequence of coinverters, conservative cartesian functor).

Proof By Corollary 3.3 applied to $p$, which is an opfibration, and then an isofibration, in Cat $/ B, p$ is conservative in Cat $/ B$, hence in $\operatorname{Fib}(B)$. Then the thesis follows from Theorem 4.5 (ii).

Acknowledgements The authors would like to thank the anonymous referee for many helpful suggestions and comments that largely improved the manuscript.

Funding Open access funding provided by Università degli Studi di Palermo within the CRUI-CARE Agreement.

Open Access This article is licensed under a Creative Commons Attribution 4.0 International License, which permits use, sharing, adaptation, distribution and reproduction in any medium or format, as long as you give appropriate credit to the original author(s) and the source, provide a link to the Creative Commons licence, and indicate if changes were made. The images or other third party material in this article are included in the article's Creative Commons licence, unless indicated otherwise in a credit line to the material. If material is not included in the article's Creative Commons licence and your intended use is not permitted by statutory regulation or exceeds the permitted use, you will need to obtain permission directly from the copyright holder. To view a copy of this licence, visit http://creativecommons.org/licenses/by/4.0/.

\section{References}

1. Cigoli, A.S., Metere, G.: Extension theory and the calculus of butterflies. J. Algebra 458, 87-119 (2016)

2. Cigoli, A.S., Mantovani, S., Metere, G., Vitale, E.M.: Fibered aspects of Yoneda's regular spans. Adv. Math. 360, 106899 (2020)

3. Giraud, J.: Méthode de la descente. Mémoires de la S. M. F. 2, VIII + 150 (1964)

4. Gray, J.W.: Fibred and cofibred categories. In: Proceedings of the Conference Categorical Algebra at La Jolla, pp. 21-83. Springer, Berlin (1966)

5. Hermida, C.: Some properties of Fib as a fibred 2-category. J. Pure Appl. Algebra 134, 83-109 (1999)

6. Johnstone, P.T.: Fibrations and partial products in a 2-category. Appl. Categ. Struct. 1, 141-179 (1993)

7. Joyal, A.: The Theory of Quasi-Categories and Its Applications, Lecture Notes, Simplicial Methods in Higher Categories at CRM Barcelona (2008)

8. Kelly, G.M.: Elementary observations on 2-categorical limits. Bull. Austral. Math. Soc. 39, 301-317 (1989)

9. Kock, A.: Monads for which structures are adjoint to units. J. Pure Appl. Algebra 104, 41-59 (1995)

10. Street, R.: Fibrations and Yoneda's lemma in a 2-category. In: Category Seminar, Sydney 1972/73, Lecture Notes in Mathematics, vol. 420, pp. 104-133. Springer, Berlin (1974)

11. Street, R.: Limits indexed by category-valued 2-functors. J. Pure Appl. Algebra 8, 149-181 (1976)

12. Street, R.: Fibrations in bicategories. Cah. Topol. Géom. Différ. 21(2), 111-160 (1980)

13. Street, R.: Categories in categories, and size matters. High. Struct. 1, 225-270 (2017)

14. Street, R., Verity, D.: The comprehensive factorization and torsors. Theory Appl. Categ. 23, 42-75 (2010)

15. Street, R., Walters, R.F.C.: The comprehensive factorization of a functor. Bull. Am. Math. Soc. 79(5), 936-941 (1973)

16. Weber, M.: Yoneda structures from 2-toposes. Appl. Categ. Struct. 15, 259-323 (2007)

17. Yoneda, N.: On Ext and exact sequences. J. Fac. Sci. Univ. Tokyo 8, 507-576 (1960)

Publisher's Note Springer Nature remains neutral with regard to jurisdictional claims in published maps and institutional affiliations. 